\title{
Uma alternativa de exodontia minimamente traumática em pacientes que fazem uso
}

\section{de bisfosfonatos}

\author{
An alternative to minimally traumatic tooth extraction in patients using bisphosphonates \\ Una alternativa a la exodoncia mínimamente traumática en pacientes que utilizan bisfosfonatos
}

Recebido: 07/02/2022 | Revisado: 16/02/2022 | Aceito: 21/02/2022 | Publicado: 03/03/2022

Enya Laissah Freire Ribeiro

ORCID: https://orcid.org/0000-0002-8087-4482 Faculdade Florence, Brasil

E-mail: enyalfreire@gmail.com

Priscilla Maria Fernandes Abdala de Alencar

ORCID: https://orcid.org/0000-0002-8341-0616

Hospital de Câncer do Maranhão Dr. Tarquínio Lopes Filho, Brasil

E-mail: priscilla-abdala@hotmail.com

\begin{abstract}
Resumo
Majoritariamente empregados na terapêutica de indivíduos portadores de neoplasias malignas, ou doenças ósseas, o grupo dos bisfosfonatos, cujo mecanismo está relacionado às propriedades antiosteoclásticas, promovem alterações na capacidade de remodelação óssea, gerando a ocorrência de um quadro de manejo desafiador: a osteonecrose dos maxilares. O presente estudo tem como objetivo de expor uma alternativa de manejo minimamente traumático de exodontia, com o fim de evitar a osteonecrose dos maxilares associada ao uso desta classe de medicamentos. Trata-se de uma revisão de literatura realizada através da seleção de artigos disponíveis nas bases de dados PubMed, LILACS e Google Acadêmico, publicados entre 2008 e 2021 nas línguas inglesa e portuguesa. A literatura aponta que a ocorrência da osteonecrose dos maxilares está relacionada com a via de administração, tempo do tratamento e a dosagem do bisfosfonato, sendo a via endovenosa a mais associada com esta condição devido a sua rápida absorção pela estrutura óssea, que é uma via amplamente utilizada em pacientes submetidos ao tratamento quimioterápico, destacando procedimentos de extração dentária como o principal desencadeador desta condição. Tomando por base que este quadro ocorre principalmente devido à manipulação óssea em procedimentos de cirurgias bucais, ressalta-se a importância de um manejo conservador para que a osteonecrose dos maxilares seja evitada, sendo a esfoliação dentária com elásticos ortodônticos uma opção viável e com resultados satisfatórios descritos pela literatura.
\end{abstract}

Palavras-chave: Bisfosfonatos; Extração dentária; Tratamento conservador; Osteonecrose.

\begin{abstract}
Mostly used in the therapy of individuals with malignant neoplasms or bone diseases, the group of bisphosphonates, whose mechanism is related to anti-osteoclastic properties, promotes changes in the bone remodeling capacity, generating the occurrence of a challenging management situation: osteonecrosis of the jaws. The present study aims to expose an alternative minimally traumatic management of tooth extraction, in order to avoid osteonecrosis of the jaws associated with the use of this class of drugs. This is a literature review carried out through the selection of articles available in PubMed, LILACS and Google Scholar databases, published between 2008 and 2021 in English and Portuguese. The literature points out that the occurrence of osteonecrosis of the jaws is related to the route of administration, treatment time and the dosage of bisphosphonate, with the intravenous route being the most associated with this condition due to its rapid absorption by the bone structure, which is a route widely used in patients undergoing chemotherapy, highlighting tooth extraction procedures as the main trigger of this condition. Based on the fact that this situation occurs mainly due to bone manipulation in oral surgery procedures, the importance of conservative management is emphasized so that osteonecrosis of the jaws is avoided, with tooth exfoliation with orthodontic elastics being a viable option with satisfactory results described by the literature.
\end{abstract}

Keywords: Bisphosphonates; Dental extraction; Conservative treatment; Osteonecrosis.

\section{Resumen}

Utilizados mayoritariamente en la terapia de individuos con neoplasias malignas o enfermedades óseas, el grupo de los bisfosfonatos, cuyo mecanismo está relacionado con las propiedades antiosteoclásticas, promueve cambios en la capacidad de remodelación ósea, generando la aparición de una situación de manejo desafiante: la osteonecrosis del mandíbulas. El presente estudio tiene como objetivo exponer una alternativa de manejo mínimamente traumático de la exodoncia, con el fin de evitar la osteonecrosis de los maxilares asociada al uso de esta clase de fármacos. Se trata de una revisión bibliográfica realizada a través de la selección de artículos disponibles en las bases de datos PubMed, LILACS y Google Scholar, publicados entre 2008 y 2021 en inglés y portugués. La literatura señala que la aparición 
de osteonecrosis de los maxilares está relacionada con la vía de administración, el tiempo de tratamiento y la dosis de bisfosfonato, siendo la vía endovenosa la más asociada a esta condición debido a su rápida absorción por la estructura ósea, lo que es una vía muy utilizada en pacientes sometidos a quimioterapia, destacándose los procedimientos de extracción dental como principal desencadenante de esta patología. Partiendo de que esta situación se da principalmente por manipulación ósea en procedimientos de cirugía bucal, se enfatiza la importancia del manejo conservador para evitar la osteonecrosis de los maxilares, siendo la exfoliación dental con elásticos de ortodoncia una opción viable con resultados satisfactorios, descrito por la literatura.

Palabras clave: Bisfosfonatos; Extracción dental; Tratamiento conservador; Osteonecrosis.

\section{Introdução}

Majoritariamente empregados na terapêutica de indivíduos portadores de neoplasias malignas e doenças ósseas, como osteoporose e doença de Paget, os bisfosfonatos representam um grupo de fármacos cujo mecanismo está relacionado às propriedades antiosteoclásticas (Moraes et al., 2020; Hasegawa et al., 2017).

Propriedades estas que por sua vez irão atribuir à estrutura óssea um aspecto mais compacto, por inibirem a remodelação óssea alterando seu turnouver, além de contribuírem com a diminuição da vascularização local (Jesus et al., 2020). Sendo estes, determinantes no agravo de uma complicação de manejo clínico desafiador relacionada ao seu uso, a osteonecrose dos maxilares associada ao uso de medicamentos (Moraes et al., 2020).

Esta osteopatologia, de etiologia ainda não esclarecida, está relacionada com o uso prolongado dos bisfosfonatos. Sendo o fármaco, tempo de uso e via de administração fatores que irão aumentar ou diminuir a ocorrência de osteonecrose, que aliados à necessidade de intervenções cirúrgicas na cavidade bucal, como exodontias, instalações de implantes, ou até mesmo trauma local, levariam a esta condição (Aguiar et al., 2021).

A osteonecrose dos maxilares é caracterizada pela presença de tecido ósseo avascular e necrótico, podendo apresentar ou não sequestros ósseos. Possuindo predileção pela mandíbula, a sintomatologia álgica e eritema podem estar presentes, todavia, quando ausentes, o diagnóstico precoce se mostra dificultado (Cunha et al., 2019).

Afim de prevenir a osteonecrose dos maxilares, é de grande relevância que o paciente que será submetido ao tratamento com bisfosfonatos realize uma adequação do meio bucal com o cirurgião-dentista antes do início do tratamento. Esta adequação tem como objetivo eliminar todo e qualquer foco de infecção odontológica, sendo esta conduta, crucial na prevenção deste quadro clínico (Khan et al., 2017).

Um protocolo de tratamento padrão para a osteonecrose dos maxilares ainda não foi estabelecido pela literatura científica. Corriqueiramente são empregadas terapias cirúrgicas ou métodos minimamente invasivos envolvendo debridamento local com soluções antibióticas, melhora na higiene bucal e antibioticoterapia sistêmica (Khan et al., 2017).

Tomando por base que o comprometimento da remodelação óssea relacionada ao uso de bisfosfonatos resultaria em prejuízo do processo de reparo alveolar, e pelo fato da ocorrência deste quadro estar frequentemente associada às cirurgias de exodontias, o manejo não cirúrgico de dentes comprometidos vem sendo uma alternativa para evitar esta condição (Silva et al., 2020).

Sendo a esfoliação com elásticos ortodônticos, uma alternativa que a literatura científica vem descrevendo resultados satisfatórios. Uma vez que esta técnica não acarretaria em danos ao tecido ósseo, somente ao ligamento periodontal (Regev et al., 2008).

Diante do exposto, o presente estudo foi realizado devido ao grande número de indivíduos que se beneficiam dessa classe de medicamentos, que representam uma relevância significativa na conduta odontológica. Sendo o objetivo deste artigo expor uma alternativa de manejo minimamente traumático de exodontia, com o fim de evitar a osteonecrose dos maxilares associada ao uso destes medicamentos. 


\section{Metodologia}

O presente estudo trata-se de uma revisão de literatura do tipo narrativa, elaborada através de uma vasta revisão nas bases de dados PubMed, Literatura Latino Americana e do Caribe em Ciências da Saúde (LILACS) e Google Acadêmico, incluindo artigos atuais e clássicos do ano de 2008 a 2021. Os artigos foram encontrados no modo "pesquisa avançada", usando um cruzamento com os seguintes descritores nas línguas portuguesa e inglesa: "Bisfosfonatos", "Bisphosphonates", "Extração dentária", "Tooth extraction", "Osteonecrose", "Osteonecrosis", "Tratamento conservador" e "Conservative treatment".

Após a busca nas bases de dados, os estudos foram filtrados mediante os seguintes critérios de exclusão: Artigos que não se referem à temática e artigos que só contenham resumos. Após seleção dos trabalhos, foi realizada uma análise extensa da literatura científica selecionada, voltada para obter os objetivos do estudo.

\section{Resultados e Discussão}

Os bisfosfonatos representam uma classe de medicamentos amplamente empregados desde $1960 \mathrm{em}$ pacientes com doenças ósseas, como osteoporose e doença de Paget, ou neoplasias malignas, como câncer de mama ou próstata (Sales \& Conceição, 2020). Sendo estes, indicados com o objetivo de prevenir metástases ósseas, neoplasias ósseas ou como tratamento de osteoporose (Oliveira et al., 2021; Eguia et al., 2020; Maciel et al., 2020).

Seu mecanismo de ação é baseado na inativação, ou indução de morte das células osteoclásticas por apoptose, resultando na inibição da reabsorção óssea que, gradativamente, irá gerar um arcabouço ósseo compacto de baixa vascularização (Venâncio et al., 2019).

Estes medicamentos são divididos em duas classes: Nitrogenados e Não Nitrogenados. Sendo os nitrogenados os mais potentes em decorrência de sua afinidade com as moléculas de nitrogênio combinadas com cálcio presentes no osso (Dorigan et al., 2021).

Sua afinidade à estrutura óssea promove um efeito acumulativo, que apresenta predileção a zonas de atividade osteoclásticas alterando o metabolismo ósseo. Culminando assim, para uma variedade de efeitos adversos, sendo a osteonecrose dos maxilares, de grande relevância na prática odontológica por ser um quadro debilitante de manejo desafiador que possui íntima relação com procedimentos cirúrgicos realizados na odontologia (Dorigan et al., 2021).

Estudos demonstram a variação da incidência deste quadro de acordo com a via de administração dos bisfosfonatos, apontando agentes antirreabsortivos orais como a via de administração que menos predispõe à ocorrência de osteonecrose. Sendo esta via, a mais empregada em pacientes com osteoporose, destacando o Aledronato como um dos mais utilizados neste perfil de paciente (Petrovic et al., 2018; Lechner et al., 2021).

Já a via de administração endovenosa, que é frequentemente utilizada em pacientes oncológicos, recebe destaque por ser mais potente que os bisfosfonatos administrados por via oral e por possuírem uma meia-vida que varia de 1-10 anos. Sendo assim, os cuidados para evitar a osteonecrose dos maxilares devem continuar mesmo após o fim do tratamento com estes medicamentos (Petrovic et al., 2018; Soutome et al., 2021).

De acordo com a Associação Americana de Cirurgiões Orais e Maxilofaciais (AAOMS), a condição da osteonecrose dos maxilares associada ao uso de medicamentos deve ser confirmada na presença dos seguintes fatores: Tratamento atual ou história de tratamento com agentes antirreabsortivos ou antiangiogênicos; Presença de fístula intra-oral ou extra-oral; Exposição de estrutura óssea por mais de 8 semanas sem remissão e paciente sem histórico de realização de radioterapia nos maxilares ou doença metastática dos maxilares (Ruggiero et al., 2014).

Possuindo estágios que irão determinar sua gravidade, bem como aspecto clínico e tratamento. Iniciando-se pelo estágio 0 , que não manifesta sinais clínicos de sequestro ósseo e possui sintomas inespecíficos. Já no estágio 1 podemos 
observar a presença de osso necrótico exposto sem presença de processo infeccioso, enquanto no estágio 2 observa-se infecção. E por fim, o estágio 3 clinicamente se manifesta com sequestros ósseos, infecção e sintomatologia dolorosa (Paiva et al., 2021; Ruggiero et al., 2014).

Como exposto na Tabela 1, extrações dentárias são amplamente apontadas como um grande fator de risco para o desenvolvimento deste quadro, assumindo assim a importância do tratamento odontológico prévio ao início do uso de bisfosfonatos com finalidade de realizar todos os procedimentos necessários para evitar intercorrências futuras relacionadas a necessidade de realização de exodontias (Hoefert et al., 2014).

Tabela 1 - Pacientes que desenvolveram Osteonecrose dos Maxilares Associada ao uso de Medicamentos após exodontias divididos por gênero, idade, patologias pregressas, agente farmacológico e via de administração utilizada.

\begin{tabular}{llllll}
\hline Autores & Gênero & Idade & Patologia pregressa & Bisfosfonato & Via de administração \\
\hline Jesus AP et al., & Feminino & 63 & Mieloma múltiplo & Zometa & Endovenosa \\
Jesus AP et al., & Masculino & 83 & Câncer de próstata & Zometa & Endovenosa \\
Jesus AP et al., & Feminino & 65 & Câncer de mama & Zometa & Endovenosa \\
Santos W et al., & Feminino & 67 & Osteoporose & Aledronato & Via oral \\
Aguiar JF et al., & Feminino & 65 & Osteoporose e câncer de mama & Aledronato & Via oral \\
\hline
\end{tabular}

Fonte: Autores

Todavia, quando os pacientes apresentam indicação de extrações dentárias durante o tratamento com bisfosfonatos, a escolha por um procedimento não-cirúrgico e conservador deve ser fortemente levada em consideração (Hoefert et al., 2014). Regev, Lustmann e Nashef (2008) propuseram uma alternativa de exodontia minimamente traumática para este grupo de pacientes, que se baseou no uso de elásticos ortodônticos com finalidade de promover a extrusão dentária.

A técnica utilizada por Regev et al. (2008) foi aplicada em 10 pacientes que fazem uso de bisfosfonatos com necessidade de realizar extrações dentárias. Baseou-se em adaptar elásticos ortodônticos ao nível da face cervical do dente que possuía indicação exodôntica. O mecanismo da técnica descrita tinha como objetivo promover o deslizamento dos elásticos em direção ao ápice do dente, conforme a proximidade com o ápice aumentava, o ligamento periodontal sofreria injúria, assim promovendo a extrusão dentária sem necessidade de manipulação óssea.

Figura 1 - Dentes após esfoliação com elásticos ortodônticos.

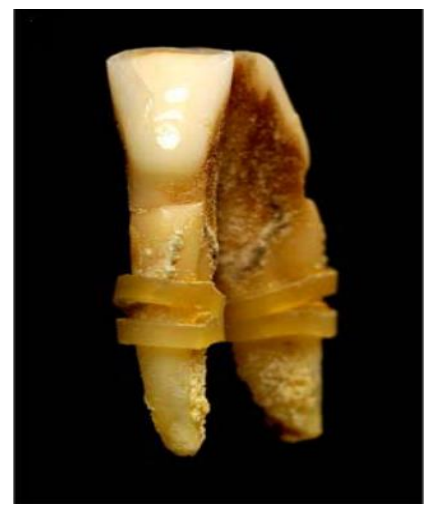

Fonte: Hoefert et al. (2014). 
Um elástico novo seria aplicado a cada semana para que o anterior fosse impulsionado em direção ao perímetro apical. Em casos de dentes multirradiculares, um elástico seria depositado em cada raiz após a divisão das mesmas, sendo que em casos de dentes vitais, o tratamento endodôntico seria realizado previamente (Regev et al., 2008).

O estudo de Regev et al. (2008) apresentou resultados satisfatórios no que se refere à ausência de intercorrências no processo de cicatrização ou exposição óssea de acordo com o acompanhamento pós-operatório, obtendo cicatrização dos tecidos moles 2 semanas após a completa esfoliação, bem como, apresentou limitações referentes a possíveis fraturas coronárias, dificuldade na esfoliação de dentes com morfologia radicular irregular e tempo médio de 6 semanas para total remoção do dente.

Como descrito na Tabela 2, Hoefert et al. (2014) realizaram um estudo aplicando a técnica utilizada por Regev et al. (2008) em três pacientes que fazem uso de bisfosfonatos. Utilizou elásticos ortodônticos tamanho $1 / 8$ e os pacientes receberam orientações quanto ao controle da infecção periodontal durante o período de esfoliação com bochechos de clorexidina $0,12 \%$, chá verde, escovação copiosa dos dentes e acompanhamento clínico.

Tabela 2 - Pacientes que fazem uso de bisfosfonatos submetidos à exodontias com elásticos ortodônticos. Divididos por patologias, via de administração do bisfosfonatos, tempo de esfoliação e presença de complicações.

\begin{tabular}{|c|c|c|c|c|c|}
\hline Autores & Mostra & Via de administração & Patologia & Tempo de esfoliação & Complicações \\
\hline Hoefert $S$, et al & 3 & Endovenosa:3 & $\begin{array}{l}\text { Tumor de Pancoast: } 1 \\
\text { Câncer de próstata: } 1 \\
\text { Câncer de mama: } 1\end{array}$ & 4.2 meses & 1 \\
\hline Regev E, et al & 10 & $\begin{array}{l}\text { Endovenosa: } 8 \\
\text { Via oral: } 2\end{array}$ & $\begin{array}{l}\text { Câncer de mama:6 } \\
\text { Mieloma múltiplo:2 } \\
\text { Osteoporose:2 }\end{array}$ & 5.8 semanas & 0 \\
\hline
\end{tabular}

Fonte: Autores.

Expondo resultados satisfatórios em dois casos, por evitar a manipulação de tecido ósseo, se resumindo apenas à reação de corpo estranho do ligamento periodontal, que reagiu formando um tecido de granulação em torno da porção radicular, promovendo a extrusão dentária sem expor estrutura óssea, garantindo um reparo favorável. Os dois casos que apresentaram resultados satisfatórios clinicamente revelaram mobilidade moderada, sinais de perda óssea, e um deles, osteonecrose dos maxilares estágio 2 com ausência de dor. Todavia, o terceiro caso apresentou complicações, pois a paciente apresentava previamente um quadro de osteonecrose dos maxilares associada ao uso de medicamentos estágio 2 com sintomatologia dolorosa, que foi controlada com debridamento, bochechos com clorexidina $0,12 \%$ e antibiótico sistêmico, porém apresentou necessidade de remover os elásticos em decorrência de sintomatologia álgica (Hoefert et al., 2014).

Apontando esta técnica como uma possível alternativa para pacientes com osteonecrose dos maxilares associada ao uso de medicamentos sem sintomatologia dolorosa, ou pacientes submetidos ao tratamento quimioterápico com bisfosfonatos sem imunossupressão significativa (Hoefert et al., 2014).

Apesar desta técnica aplicada em pacientes que fazem uso de bisfosfonatos ter sido descrita pela primeira vez no estudo de Regev et al. (2008), o uso de elásticos ortodônticos para realização de exodontia minimamente traumática já havia sido empregado em pacientes com hemofilia (Hoefert et al., 2014).

O conceito da realização de exodontias minimamente invasivas vem sendo cada vez mais discutido quanto às suas inúmeras vantagens quando aplicado em pacientes que fazem uso de bisfosfonatos, sendo esta uma alternativa que visa evitar a osteonecrose dos maxilares. Apresentando características importantes na garantia da preservação alveolar e rebordo ósseo, que, corriqueiramente em exodontias convencionais, sofreriam injúria num nível maior (Silva et al.,2020). 


\section{Considerações Finais}

Grande parte da população faz uso de forma recorrente de medicamentos da classe dos bisfosfonatos, que possuem mecanismos específicos que inibem a reabsorção óssea, assim promovendo alterações na sua remodelação. Dentre suas reações adversas, a de maior significado na odontologia é a osteonecrose dos maxilares, uma vez que estes medicamentos atribuem à estrutura óssea pouca vascularização local, adquirindo uma natureza mais compacta, consequentemente, prejudicando o reparo alveolar.

A etiologia desta condição ainda não foi totalmente estabelecida, podendo se manifestar tanto em decorrência de trauma local, quanto espontaneamente. Sendo os procedimentos de instalações de implantes e exodontias, os mais relacionados com a osteonecrose dos maxilares. Devido ao seu manejo ser considerado um desafio, a adequação do meio bucal antes do início da terapia com bisfosfonatos é indicado para evitar intercorrências maiores durante seu uso.

A via de administração, tipo de fármaco e tempo de uso são fatores que devem ser levados em consideração, pois estes estão diretamente ligados à ocorrência da osteonecrose dos maxilares, sendo a via endovenosa, a mais relacionada com esta complicação, possuindo um grande potencial acumulativo na estrutura óssea durante anos.

Quando há necessidade de realizar cirurgias orais, como exodontias, em pacientes que estejam fazendo o uso ativo destas medicações, é fortemente indicado que o cirurgião-dentista opte por realizar procedimentos minimamente invasivos. De acordo com a literatura científica, a realização de procedimentos de forma minimamente traumática, mostra-se atrativa.

Dentre as alternativas, destaca-se a utilização de elásticos ortodônticos para realizar extrações dentárias em pacientes submetidos a esta terapêutica. Estudos que realizaram esta técnica apresentaram resultados satisfatórios em sua maioria e vantagens por ser um método de fácil realização e por causar injúria somente ao ligamento periodontal, assim preservando a estrutura alveolar, apesar de necessitarem de um espaço de tempo maior para completa esfoliação e colaboração do paciente.

Embora os artigos encontrados apresentem resultados promissores, nota-se a presença de lacunas na literatura referentes ao uso de elásticos ortodônticos com finalidade de exodontia, assim reforçando a necessidade de realizações de futuras pesquisas, para que assim sejam obtidos maiores níveis de evidência científica.

\section{Referências}

Aguiar, J. F., Ferreira, J. S., França, S. R., Martins, M., Guimarães, H., Eduardo, A., Sampieri, M. (2021). Tratamento cirúrgico de osteonecrose induzida por medicamentos após falha de tratamento conservador: relato de caso. Brazilian Journal of Health Review, 4(1):3108-3115.

Cunha, K., Lippert, V., Baldino, M., Dreyer, J., Cunha, R. (2019). Alternativa de tratamento reabilitador para pacientes em uso de bisfosfonatos intravenoso. Revista Odontológica de Araçatuba, 40(1):29-34.

Dorigan, M. C., Matias, J. B., Tognetti, V. M., Torres, S. (2021). A osteonecrose dos maxilares induzida por bisfosfonatos: uma revisão de literatura. J Research, Society and Development, 10(16), e92101623466.

Eguia, A., Bagan, L., Cardona, F. (2020). Review and update on drugs related to the development of osteonecrosis of the jaw. Med Oral Patol Oral Cir Bucal, 25(1):71-83.

Hasegawa, T., Kawakita, A., Ueda, N., Funahara, R., Tachibana, A., Kobayashi, M., Kondou, E., Takeda, D., Kojima, Y., Sato, S., Yanamoto, S., Komatsubara, H., Umeda, M., Kirita, T., Kurita, H., Shibuya, Y., Komori, T. (2017). A multicenter retrospective study of the risk factors associated with medication-related osteonecrosis of the jaw after tooth extraction in patients receiving oral bisphosphonate therapy: can primary wound closure and a drug holiday really prevent MRONJ? Osteoporosis International. 28:2465-247.

Hoefert, S., Grimm, M., Sharghi, F., Geist, A., Krimmel, M., Reinert, S. (2014). Atraumatic tooth extraction in patients taking bisphosphonates: a review of literature and experience with three cases. Oral Maxillofac Surg, 18(3):341-349.

Jesus, A. P., Sousa, F., Cardoso, J. A., Cancio, A.V., Simões, C., Farias, J. (2019). Tratamento cirúrgico para osteonecrose dos maxilares induzida por bisfosfonatos: relatos de casos. RFO UFP, 24(1):22-30.

Khan, A. A., Morrison, A., Kendler, D. L., Rizzoli, R., Hanley, D. A., Felsenberg, D., Mccauley, L., O’Ryan, F., Reid, I., Ruggiero, S., Taguchi, A., Tetradis, S., Watts, N., Brandi, M., Peters, E., Guise, T., Eastell, R., Cheung, A., Morin, S. ... Compston, J. (2017). Case-Based Review of Osteonecrosis of the Jaw (ONJ) and Application of the International Recommendations for Management From the International Task Force on ONJ. J. Clin. Densitom, 20(1):8-24. 
Research, Society and Development, v. 11, n. 3, e45211326683, 2022

(CC BY 4.0) | ISSN 2525-3409 | DOI: http://dx.doi.org/10.33448/rsd-v11i3.26683

Lechner, J., Von Baehr, V., Zimmermann, B. (2021). Osteonecrosis of the jaw beyond bisphosphonates: Are there any unknown local risk factors? Clinical, Cosmetic and Investigational Dentistry, 13:21-37.

Maciel, A. P., Quispe, R. A., Martins, L. J., Caldas, R. J., Santos, P. S. (2020). Clinical profile of individuals with bisphosphonate-related osteonecrosis of the jaw: An integrative review. Med J, 138(4):326-35.

Moraes, C. M., Oliveira D.C., Favretto C.O. (2020). Osteonecrose dos maxilares associada ao uso dos bisfosfonatos: revisão de literatura. Saúde Multidisciplinar (Mineiros),8(2):45-50.

Oliveira, C., Dantas, J. B., Borges, D. P., Martins, G. B., Medrado, A. R., Reis, J. V., Marchionni, A. M. (2021). Ozonotherapy for Treatment of Radiation Therapy-induced Jaw Osteonecrosis and Bisphosphonates: Case Report. Revista Brasileira de Cancerologia, 67(2):1-5.

Paiva, C., Silva, N. M., Oliveira, T. A., Souza, J. A., Pereira, G. M., Junior, F. A. (2021). Osteonecrose dos maxilares relacionada ao uso de medicamentos: revisão sistemática. J Research, Society and Development, 10(5), e15510514740.

Petrovic, I., Rosen, E. B., Matros, E., Huryn, M., Shah, J. P. (2018). Oral rehabilitation of the cancer patient: a formidable challenge. J Surg Oncol,117(8):1729-35.

Regev, E., Lustmann, J., \& Nashef, R. (2008). Atraumatic Teeth Extraction in Bisphosphonate-Treated Patients. J Oral Maxillofac Surg, 66(6):1157-61.

Ruggiero, S. L., Dodson, T. B., Fantasia, J., Goodday, R., Aghaloo, T., Mehrotra, B., O’ Ryan, F. (2014). American association of oral and maxillofacial surgeons position paper on medication-related osteonecrosis of the jaw - 2014 update. J Oral Maxillofac Surg, 72(10):1938-56.

Silva, L. F., Silva, L. A., Silva, G. G., Paiva, D., Lira, K. B., Pinheiro, J. (2020). Conceitos Atuais Em Exodontia Atraumática: revisão de literatura. Revista de Odontologia da Braz Cubas, 10(1):34-45.

Sales, K. O., Conceição L. S. (2020). A atuação do cirurgião-dentista frente à osteonecrose dos maxilares associada ao uso de bisfosfonatos: uma revisão de literatura. Rev. Facit Bus Technol J, 14(2):99-110.

Soutome, S., Otsuru, M., Hayashida, S., Murata, M., Yanamoto, S., Sawada, S., Kojima, Y., Funahara, M., Iwai, H., Umeda, M., Saito, T. (2021). Relationship between tooth extraction and development of medication-related osteonecrosis of the jaw in cancer patients. Sci Rep,11(1):1-8.

Venâncio, R., Dantas, F., Euzébio, R. (2019). Terapia fotodinâmica como coadjuvante no tratamento da osteonecrose dos maxilares associada ao uso de medicamentos (OMAN). SALUSVITA, 38(4):1093-1105. 